\title{
Utilização de Testes para Avaliar Crianças dos 0 aos 7 anos: Resultados de um Inquérito a Psicólogos Portugueses
}

\author{
Use of Tests to Assess Children between 0 and 7 Years Old: Results of an Inquiry \\ Addressed to Portuguese Psychologists
}

\author{
Maria João Seabra-Santos ${ }^{1}$, Mário R. Simões ${ }^{2}$, Pedro Armelim Almiro ${ }^{3}$ e Leandro S. Almeida ${ }^{4}$
}

\begin{abstract}
Resumo
Sendo a avaliação psicológica e o recurso a testes atividades identitárias dos psicólogos, estudos nacionais e internacionais têm destacado um menor investimento quando se trata de avaliar crianças nos primeiros anos de vida. Esta investigação tem como objetivo caracterizar a utilização de testes e outros instrumentos de avaliação psicológica com crianças com 0-7 anos em Portugal. A amostra é composta por 63 psicólogos que responderam a um inquérito online no âmbito de um estudo mais vasto. A análise dos instrumentos referidos como mais usados permitiu ressaltar que estes são sobretudo testes (e menos inventários) e que a área cognitiva está mais representada do que a socio-emocional, sendo as escalas de Wechsler WISC-III e WPPSI-R e as Escalas de Desenvolvimento Mental de Griffiths os instrumentos mais mencionados. A discussão dos resultados remete para a necessidade de mais investigação no sentido de garantir a qualidade psicométrica dos instrumentos utilizados, e de um forte investimento na formação dos profissionais nesta área.
\end{abstract}

Palavras-chave: avaliação psicológica; testes psicológicos; idade pré-escolar; crianças 0-7 anos

\begin{abstract}
With psychological assessment and testing being part of psychologists' identity, Portuguese and international studies have highlighted less investment in assessing children during their early years. This research aims to characterize the use of testing and other psychological assessment tools on children between 0-7 years old in Portugal. The sample is composed of 63 psychologists who answered an online inquiry within the scope of a broader study. The analysis of instruments most used allowed to reinforce that these mainly consist of tests (and less of rating scales), and also that the cognitive field is more represented than the social-emotional one, with the Wechsler WISC-III and WPPSI-R scales and the Griffiths Mental Development Scales being the most frequently mentioned instruments. The discussion of the results points to the need of further investigation aimed at guaranteeing the psychometric quality of instruments used, and of significant investment in training the field's professionals.
\end{abstract}

Keywords: psychological assessment, psychological tests, pre-school age, children 0-7 years old

Agradecimentos: Os autores estão gratos à Ordem dos Psicólogos Portugueses (OPP) pelo envio aos psicólogos portugueses de um convite à sua participação no estudo.

\footnotetext{
${ }^{1}$ Doutora em Psicologia. Professora Auxiliar na Universidade de Coimbra. Universidade de Coimbra, CINEICC - Centro de Investigação em Psicologia e Intervenção Cognitivo-Comportamental, Faculdade de Psicologia e de Ciências da Educação, PsyAssessmentLab. Rua do Colégio Novo, 3000-115 Coimbra, Portugal. Tel.: 00351 239851450. E-mail: seabramj@fpce.uc.pt

2 Doutor em Psicologia. Professor Catedrático na Universidade de Coimbra. Universidade de Coimbra, CINEICC - Centro de Investigação em Psicologia e Intervenção Cognitivo-Comportamental, Faculdade de Psicologia e de Ciências da Educação, PsyAssessmentLab. Rua do Colégio Novo, 3000-115 Coimbra, Portugal.Tel.: 00351 239851450. E-mail: simoesmr@fpce.uc.pt

3 Doutor em Psicologia. Professor Auxiliar na Universidade Autónoma de Lisboa, , CIP - Centro de Investigação em Psicologia, CINEICC Centro de Investigação em Neuropsicologia e Intervenção Cognitivo-Comportamental, Departamento de Psicologia, PsyAssessmentLab. Rua de Santa Marta, 47, 1150-293 Lisboa, Portugal. Tel.: 00351 213177600. Email: paalmiro@ autonoma.pt

4 Doutor em Psicologia. Professor Catedrático na Universidade do Minho Universidade do Minho; CIED - Centro de Investigação em Educação; Instituto de Educação. . Campus de Gualtar, 4710-057 Braga, Portugal. Tel.: 00351 253604255. E-mail: leandro@ie.uminho.pt

Revista Iberoamericana de Diagnóstico y Evaluación - e Avaliação Psicológica. RIDEP · N60 · Vol.3 · 81-94 · 2021

ISSN: 1135-3848 print /2183-6051online
} 


\section{Introdução}

$\mathrm{Na}$ sessão de abertura da European Conference of Psychological Assessment (Lisboa, julho de 2017), o então presidente da European Association of Psychological Assessment, Johnny Fontaine, defendeu a importância da avaliação psicológica enquanto campo de estudo da Psicologia, tanto numa perspetiva teórica, como prática. Com efeito, é através da avaliação que as teorias psicológicas podem ser testadas empiricamente, refinadas ou refutadas $\mathrm{e}$ as intervenções em Psicologia podem ser validadas com base em evidências (Fontaine, 2017). Na verdade, desde o seu surgimento que a avaliação psicológica (que inclui o recurso a testes validados) é considerada como uma área identitária dos psicólogos, integrando o núcleo das suas competências específicas ao nível da prática, formação e investigação (Wright et al., 2017).

No que diz respeito à prática profissional, a avaliação psicológica constitui um domínio privilegiado de atuação do psicólogo, que cruza diversas áreas (Ricou et al., 2018). Assim, em contexto escolar, clínico, forense ou organizacional, o psicólogo é chamado a avaliar e o momento da avaliação psicológica constitui, em geral, o ponto de partida para a caracterização da situação, para o início do processo de intervenção ou, ainda, para a avaliação da eficácia do mesmo (Cohen \& Swerdlik, 2018).

Neste contexto, a avaliação psicológica nos primeiros anos de vida reveste-se de particular importância. As evidências científicas reforçam o papel determinante que as experiências ocorridas nesta fase da vida têm para o curso do desenvolvimento (National Scientific Council on the Developing Child, 2004). Dada a grande plasticidade do ponto de vista neurobiológico, a criança encontra-se particularmente vulnerável a fatores de risco, mas também recetiva à mudança num sentido positivo (Carvalho et al., 2016). Assim, mesmo crianças que enfrentam fatores de adversidade significativos, são capazes de desenvolver competências fundamentais para a vida, desde que as políticas, programas e cuidadores proporcionem meios suficientemente apoiantes para o seu desenvolvimento (Center on the Developing Child at Harvard University, 2017). O objetivo da avaliação psicológica será, pois, compreender cada criança na sua individualidade, monitorizar o seu desenvolvimento e planificar estratégias interventivas, caso se identifique alguma necessidade específica (Washington State Office of Superintendent of Public Instruction, 2008). A abordagem a adotar deve ser compreensiva, envolver a participação dos pais e educadores no contexto de um modelo colaborativo, e usar múltiplos métodos, ajustados à individualidade de cada caso.

Porém, se hoje em dia as vantagens de uma intervenção precoce estão claramente mais assimiladas, a sua implementação é muitas vezes travada pela falta de ferramentas de despistagem e avaliação que captem os problemas mais comuns nesta faixa etária (Barbarin, 2007). Na verdade, se esta escassez é notória em diversas áreas da avaliação psicológica e para diversas idades, ela é mais saliente na avaliação de crianças nos primeiros anos de vida, faixa etária para a qual os desenvolvimentos foram desde sempre mais tardios, não só no nosso país, mas também a nível internacional (Major \& Seabra-Santos, 2014). Com efeito, até a criança entrar na escola e os problemas cognitivos ou socioemocionais se tornarem mais evidentes, prevaleceu por muito tempo a abordagem do "esperar para ver" (Breitenstein et al., 2009).

A qualidade da avaliação psicológica e a sua utilidade em termos práticos estão diretamente dependentes das propriedades dos instrumentos utilizados para a concretizar. Neste contexto, diversos investigadores e entidades com responsabilidades no domínio têm-se dedicado à elaboração de diretrizes e recomendações para a adaptação e uso de instrumentos de avaliação psicológica (American Educational Research Association et al., 2014; Archer et al., 2016; Casullo, 2009; International Test Commission, 2013, 2017; Prieto \& Muñiz, 2000). De entre as características destacadas como devendo estar presentes nestes instrumentos são comummente sublinhados a sua adequação à população alvo e aspetos contextuais, a existência de dados normativos atualizados e representativos e de estudos que atestem a respetiva precisão e validade para os fins visados. A Ordem dos Psicólogos Portugueses (OPP) acompanhou estas preocupações, ao introduzir no seu Código 
Deontológico (OPP, 2011), uma secção dedicada aos princípios específicos da avaliação psicológica onde, logo na introdução, se esclarece sobre a necessidade de esta ser concretizada através do "recurso a protocolos válidos". No contexto da OPP é também de realçar a criação de um Conselho Consultivo para a Acreditação de Testes e Provas Psicológicas (OPP, 2018).

O escrutínio dos instrumentos de avaliação psicológica usados em diversos países e em contextos variados tem sido efetuado através de inquéritos realizados junto de psicólogos, os quais têm analisado questões como as crenças e atitudes dos profissionais em relação à prática da avaliação, quais os instrumentos a que mais recorrem nesse contexto ou a sua perceção sobre a adequação técnica das medidas utilizadas. Enquanto alguns destes inquéritos incluíram todas as áreas do exercício da psicologia (Wright et al., 2017), outros focaram-se em contextos profissionais mais restritos, nomeadamente áreas que tiveram uma ascensão rápida nas últimas décadas, como é o caso da psicologia forense (LaDuke et al., 2017) ou da neuropsicologia (cf. Piotrowski, 2017, para uma revisão sistemática da literatura), ou áreas mais clássicas, como é o caso da psicologia da educação (Albritton et al., 2019; Benson et al., 2019; Oakland et al., 2016). Um inquérito recente analisou especificamente o papel de psicólogos escolares que trabalham com crianças antes da entrada na escola $(n=135) \mathrm{e}$ concluiu que pelo menos metade do seu tempo profissional é dedicado a tarefas de avaliação, tendo como foco, na maior parte dos casos, necessidades especiais dos alunos (Albritton et al., 2019). A maioria dos estudos realizados é de âmbito nacional havendo, no entanto, vários que abrangeram diversos países, nomeadamente europeus (Muñiz et al., 2001; Evers et al., 2012). O estudo conduzido por Evers e colaboradores (2017) merece destaque particular neste contexto, na medida em que envolveu 20.467 profissionais de 29 países e cinco continentes. Destas várias investigações é possível destacar um considerável consenso entre os psicólogos participantes quanto ao uso de testes no exercício da sua profissão e ao reconhecimento da utilidade destes instrumentos. Ao contribuírem para um maior conhecimento sobre o que é feito e como é entendido o trabalho da avaliação psicológica por parte de quem o pratica, estes estudos têm o potencial de evidenciar os desenvolvimentos na área $\mathrm{e}$, ao mesmo tempo, de identificar lacunas que possam guiar escolhas mais esclarecidas, ao nível individual e institucional, nomeadamente nos campos da investigação e da formação.

Também em Portugal inquéritos do mesmo tipo procuraram ciclicamente esclarecer quais as atitudes dos psicólogos face à avaliação psicológica, o tipo de práticas mais instituídas neste contexto e/ou os testes e outros instrumentos de avaliação mais utilizados (Almeida \& Cruz, 1985; Cruz \& Almeida, 1987; Diniz et al., 2007; Muñiz et al., 1999; Almeida et al., 2018). Estes estudos, realizados regularmente com uma periodicidade de cerca de 10 anos, puseram em evidência a aceitação e a importância atribuída ao processo de avaliação psicológica enquanto atividade identitária dos psicólogos, generalizada e reconhecida dentro e fora da profissão. Ao mesmo tempo, salientaram a fragilidade técnica de alguns dos instrumentos, considerados inapropriados ou desatualizados, e a consequente necessidade de maiores investimentos neste campo.

A maioria dos inquéritos referidos analisaram os instrumentos independentemente da idade a que se destinam, não existindo, tanto quanto é do nosso conhecimento, investigações nacionais que se foquem especificamente na utilização de instrumentos de avaliação psicológica nos primeiros anos de vida. O objetivo central do presente estudo consiste em caracterizar a utilização que é feita em Portugal de testes e outros instrumentos de avaliação psicológica para avaliar crianças desde o nascimento até aos 7 anos de idade. Mais especificamente, pretende-se caracterizar os psicólogos que utilizam este tipo de instrumentos do ponto de vista sociodemográfico, da formação profissional e do campo de atuação profissional. O estudo visa, igualmente, compreender quais os objetivos com que tais instrumentos são utilizados e quais são aqueles que os psicólogos mais usam, nestas idades. Os dados foram recolhidos no âmbito de um projeto internacional que envolveu diversos países iberoamericanos, incluindo Portugal (Almeida et al., 2018), projeto este do qual já resultaram algumas publicações (Seabra-Santos et al., 2019; Muñiz et al., 2020; Reppold et al., 2020). 


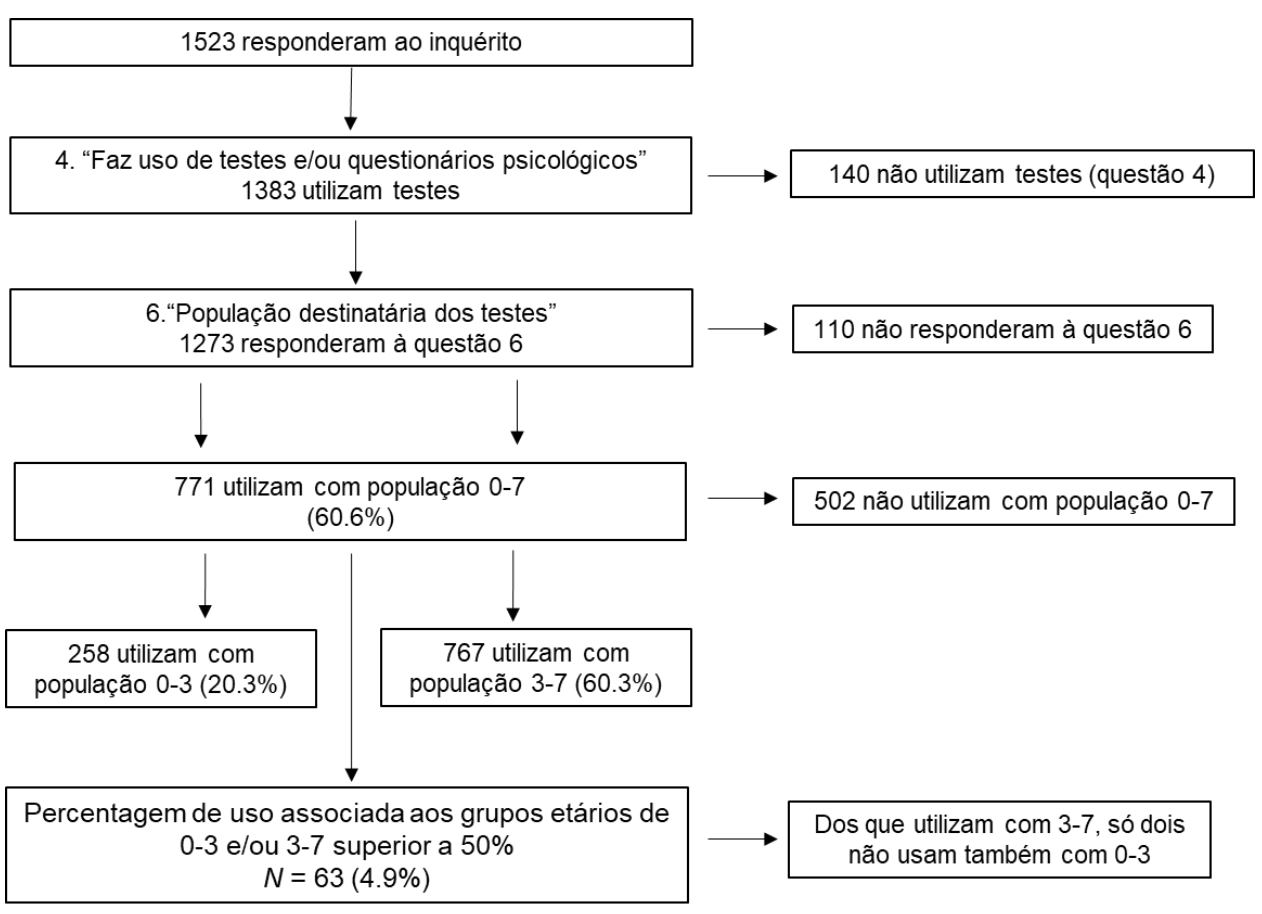

Figura 1. Fluxograma da seleção da amostra

\section{Método}

\section{Amostra}

Os participantes são psicólogos portugueses que responderam on-line a um inquérito relativo a "Atitudes e uso dos testes psicológicos nos países ibero-americanos". Embora o inquérito tenha sido realizado em vários países ibero-americanos, para o presente estudo foram considerados apenas os respondentes portugueses. A Figura 1 representa o fluxograma relativo à seleção da amostra, partindo do número total de respondentes ao inquérito, igual a 1523, dos quais $1383(90.8 \%)$ afirmaram utilizar testes e/ou questionários psicológicos, de forma habitual, no exercício da sua atividade profissional. As respostas dadas ao inquérito por esta amostra de respondentes serviram de base a uma investigação anterior, que teve como objetivo caracterizar a utilização de testes e outros instrumentos de avaliação no nosso país e identificar atitudes e problemas relacionados com esta (Seabra-Santos et al., 2019). A presente investigação baseia-se nas respostas dadas por uma subamostra dos participantes nesse estudo, tendo em conta o objetivo de centrar a análise nos respondentes que referiram usar testes para avaliar crianças nos primeiros anos de vida.

Assim, a seleção dos participantes foi feita com base nas respostas dadas à questão 6 (População destinatária dos testes), tendo sido selecionados os que referiram uma percentagem de uso superior a $50 \%$ associada aos grupos etários de 0 a 3 anos e/ou de 3 a 7 anos, sendo retidos para o presente estudo 63 participantes, o que corresponde a $4.9 \%$ dos psicólogos que responderam à questão 6 . As respostas relativas às duas faixas etárias foram analisadas em conjunto, uma vez que, dos psicólogos que responderam utilizar testes com crianças dos 0 aos 3 anos, somente dois não os usavam também com crianças dos 3 aos 7 anos. Dos 63 participantes, 56 eram do sexo feminino $(88.9 \%)$ e a maioria $(n=32 ; 50.8 \%)$ tinha completado o grau de Mestre pós-Bolonha, havendo um menor número de participantes com uma Licenciatura de cinco anos pré-Bolonha $(n=20 ; 31.7 \%)$ ou uma especialização pósBolonha $(n=8 ; 12.7 \%)$, e sendo residual o número de doutorados $(n=3 ; 4.8 \%)$. As idades oscilavam entre os 26 e os 74 anos, com uma média de 37.79 anos $(D P=8.33)$. O número de anos de atividade profissional variava entre 1 e 45 , com a moda situada nos 10 anos $(n=8)(M=12.29, D P=7.96) \mathrm{e}$ uma maior concentração de casos (36.5\%) com 10 a 13 anos de prática.

\section{Instrumento}

Para realizar o inquérito recorreu-se a um questionário elaborado para o efeito e no qual se 
adotou para as várias questões uma formulação comum que fosse o mais ajustada possível às realidades de todos os países participantes. $\mathrm{Na}$ introdução era explicado o objetivo e interesse do estudo, assim como o caráter anónimo das respostas. O questionário era composto por nove partes. $\mathrm{Na}$ primeira eram solicitados alguns elementos de caracterização (e.g., idade, sexo, país, região, nível de formação). Na segunda secção era pedido o campo de exercício da atividade profissional e na terceira a área de formação profissional sendo, em ambos os casos, dadas alternativas de resposta (e.g., Psicologia Clínica e da Saúde, Psicologia Escolar e da Educação, Psicologia do Trabalho e das Organizações, Psicologia Social e Comunitária). Nestas duas secções pedia-se a indicação de três opções, por ordem de importância.

A quarta questão, de resposta Sim/Não, indagava sobre o uso de testes e questionários no exercício da atividade profissional. A pergunta 5 focava-se nos objetivos da administração de testes de entre um conjunto de opções (e.g., Diagnóstico, Recrutamento, Orientação ou aconselhamento, Avaliação de programas). A sexta questão incidia sobre a população destinatária dos testes (e.g., 0-3 anos, 3-7 anos, 813 anos). Para as questões cinco e seis pedia-se, igualmente, a percentagem de uso associada a cada objetivo/grupo etário. A secção 7 questionava sobre quais os testes que $\mathrm{o}$ respondente mais utilizava na sua atividade profissional, podendo ser indicados até 10 instrumentos, por ordem decrescente da frequência de utilização. $\mathrm{Na}$ secção 8 eram inventariadas atitudes (e.g., No desempenho $d a$ minha profissão o uso de testes dá-me confiança) e problemas (e.g., Uso por pessoas não qualificadas) na utilização de testes, devendo o psicólogo responder se concordava ou não com cada afirmação, numa escala de 1 (total desacordo) a 5 (total acordo). Finalmente, na secção 9 disponibilizava-se um espaço no qual podiam ser acrescentados comentários acerca do uso dos testes no país do respondente. Com exceção da secção 8 (atitudes e problemas), já analisada em detalhe num artigo anterior (Seabra-Santos et al., 2019) e relativamente à qual a amostra do presente estudo não acrescenta informação diferente, são analisadas neste artigo as respostas da nossa amostra a todas as outras questões incluídas no inquérito.

\section{Procedimentos}

O projeto contou com o apoio da Comissão Internacional de Testes, da Sociedade IberoAmericana de Psicologia e de organismos profissionais nacionais. No caso português o estudo foi apoiado pela Ordem dos Psicólogos Portugueses, que enviou aos psicólogos associados um convite à sua participação. O questionário foi aplicado online sendo as respostas dos psicólogos respondentes diferenciadas pelo país. Mesmo sendo anónima a participação, salvaguardou-se a confidencialidade dos dados atendendo às questões pessoais colocadas.

As análises dos dados foram conduzidas com o programa IBM SPSS Statistics, versão 26.0 para Windows. Face aos objetivos do presente artigo, as análises circunscreveram-se a estatísticas descritivas.

\section{Resultados}

Os psicólogos que referiram utilizar testes e questionários maioritariamente para a avaliação psicológica de crianças de idades entre os 0 e os 7 anos fizeram a sua formação profissional sobretudo na área da Psicologia Clínica e da Saúde (57.1\%), seguida da Psicologia Escolar e da Educação (20.6\%), sendo o seu campo de atuação profissional também preferencialmente distribuído por estas duas áreas $(42.9 \%$ e $38.1 \%$, respetivamente). No que respeita à segunda opção indicada, as maiores percentagens continuaram a recair sobre as mesmas áreas, mas neste caso a Neuropsicologia surgiu como uma terceira área a agregar uma percentagem expressiva de participantes $(20.0 \%$ e $9.5 \%$, respetivamente para a área de formação e área de atividade profissional).

O Quadro 1 apresenta a percentagem de uso associada a cada um dos objetivos de utilização de testes sugeridos como opções no questionário. Como se pode observar, o objetivo de diagnóstico apareceu referenciado com uma maior percentagem de utilização (59.21\%), seguido pelo de rastreio (18.87\%). A avaliação de programas e a investigação surgiram associadas a percentagens menos expressivas $(7.14 \%$ e $5.90 \%$, respetivamente). Outros objetivos, como a seleção e a orientação, foram também assinalados, embora 
Quadro 1. Estatísticas descritivas das percentagens para cada objetivo de utilização

\begin{tabular}{lcccc}
\hline & Mínimo & Máximo & Média & Desvio-padrão \\
\hline Rastreio & 0 & 100 & 18.87 & 19.63 \\
Avaliação/diagnóstico & 0 & 100 & 59.21 & 26.40 \\
Seleção & 0 & 30 & 1.46 & 5.64 \\
Orientação & 0 & 50 & 6.43 & 11.89 \\
Avaliação programas & 0 & 40 & 7.14 & 10.95 \\
Investigação & 0 & 100 & 5.90 & 19.20 \\
Docência & 0 & 50 & 0.90 & 6.32 \\
Outro & 0 & 5 & 0.08 & 0.63 \\
\hline
\end{tabular}

não se possam enquadrar na avaliação da faixa etária em análise. Tal pode ser explicado pela circunstância de a maioria dos psicólogos da amostra deste estudo se dedicarem igualmente à avaliação psicológica de outras faixas etárias.

Os instrumentos referidos como sendo utilizados por mais de 5\% dos participantes, num total de 15 instrumentos, foram analisados em função de diversos critérios, sintetizados no Quadro 2. Nesta análise foram tidos em conta o número e percentagem de vezes que os instrumentos foram referidos, independentemente da ordem (recorde-se que era pedido um número de instrumentos até 10 , em ordem decrescente de utilização).

Tal como se pode observar, dos 15 instrumentos considerados, 13 são testes (dos quais quatro projetivos), nove têm como foco da avaliação construtos de natureza cognitiva e somente cinco são destinados especificamente a crianças antes da entrada na escola. Alguns destes instrumentos não dispõem de referenciais normativos e/ou de estudos de validade atualizados para a população portuguesa.

No que diz respeito aos instrumentos específicos, a WISC (edição III ou não discriminada) e a Escala de Desenvolvimento Mental de Griffiths constituíram os mais referidos em geral, com percentagens de utilizadores bastante expressivas. Estes foram também os instrumentos mais mencionados em primeiro lugar agregando, neste caso, percentagens de participantes iguais a $26.5 \%$ e a $19.1 \%$, respetivamente. A avaliação socioemocional ou da personalidade parece ser feita mais através do recurso a técnicas projetivas (CAT, Desenho da Família, Roberts ou Era uma vez...), reportadas por $40 \%$ dos respondentes, do que por meio de inventários comportamentais preenchidos por pais e educadores (Escalas de Achenbach nas suas diversas versões ou Escala de Conners), assinalados por 19\%. Por outro lado, em linha com alguma tradição na psicologia da criança, a avaliação psicológica nas áreas socioemocional e/ou cognitiva recorre a provas gráficas (desenho), nomeadamente Desenho da Família ou Desenho da Figura Humana. Finalmente, reporta-se a utilização de provas de avaliação da prontidão escolar (Provas de Diagnóstico Pré-Escolar) e de aptidões necessárias para as aprendizagens escolares (BAPAE), orientadas para a avaliação de crianças na transição do pré-escolar para o Ensino Básico.

Dos 63 psicólogos que constituíram a amostra do presente estudo, $11(17.5 \%)$ responderam à última secção do inquérito, questão aberta destinada a comentários adicionais sobre o uso de testes. Cinco destes participantes mencionaram nos seus comentários os preços elevados dos materiais, que pode ser impeditivo da respetiva aquisição. Quatro psicólogos referiram a dificuldade de acesso a testes validados, seja pelo número reduzido de testes com estudos portugueses, ou pela indisponibilidade desses materiais nas instituições (o que poderá remeter também para a questão económica), ou mesmo no mercado (como é o caso da WPPSI-R). Houve, ainda, uma resposta que focou a necessidade de mais formação sobre aplicação e interpretação dos testes, e uma outra assinalando a necessidade de mais regulamentação na área. 
Quadro 2. Instrumentos mais usados na faixa etária 0-7 anos $(N=63)$

\begin{tabular}{|c|c|c|c|c|c|c|c|c|}
\hline & Instrumento & $n$ & $\%$ & $\begin{array}{l}\text { Tipo de } \\
\text { instrumento }\end{array}$ & $\begin{array}{c}\text { Foco } \\
\text { da avaliação }\end{array}$ & $\begin{array}{l}\text { Idades de } \\
\text { aplicação } \\
\text { (em anos) }\end{array}$ & Estudos em Portugal & $\begin{array}{l}\text { Especificidade para } \\
\text { avaliar crianças até à } \\
\text { entrada na escola }\end{array}$ \\
\hline$\overline{1}$ & $\begin{array}{l}\text { WISC } \\
\text { - WISC III }(n=41) \\
\text { - WISC-R }(n=1)\end{array}$ & 42 & 67 & Testes & Inteligência & $6-16$ & $\begin{array}{l}\text { - WISC-III: adaptação, estudos normativos e de } \\
\text { validade (Wechsler/ Simões et al./CEGOC, 2003b) } \\
\text { - WISC-R: não aferido }\end{array}$ & Não \\
\hline 2 & Griffiths & 25 & 40 & Testes & $\begin{array}{l}\text { Desenvolvimento / } \\
\text { Cognitivo }\end{array}$ & $\begin{array}{c}0-2 \\
2-8 \\
0-6\left(3^{\mathrm{a} e d}\right)\end{array}$ & $\begin{array}{l}\text { - Griffiths 0-2 e 2-8: tradução (Griffiths et } \\
\text { al./Ferreira et al./CEGOC, 2007) } \\
\text { - Griffiths 2-8: estudos normativos preliminares } \\
\text { (Borges et al., 2012) } \\
\text { - Griffiths-III 0-6: tradução (Stroud et al./Ferreira et } \\
\text { al./Hogrefe, 2018) }\end{array}$ & Sim \\
\hline 3 & $\begin{array}{l}\text { WPPSI } \\
\text { - WPPSI-R }(n=15) \\
\text { - WPPSI-IV }(n=1)\end{array}$ & 16 & 25 & Testes & Inteligência & $3-6^{1 / 2}$ & $\begin{array}{l}\text { - WPPSI-R: adaptação, estudos normativos e de } \\
\text { validade (Wechsler/Seabra-Santos et al./CEGOC, } \\
\text { 2003a) } \\
\text { - WPPSI-IV: não aferido }\end{array}$ & Sim \\
\hline 4 & $\begin{array}{l}\text { Matrizes Progressivas } \\
\text { Coloridas de Raven }\end{array}$ & 15 & 24 & Teste & Raciocínio & $5-$ & $\begin{array}{l}\text { - CPM: estudos normativos e de validade (Simões, } \\
\text { 2000) } \\
\text { - CPM-P: estudos normativos e de validade } \\
\text { (CEGOC, 2009) }\end{array}$ & Não \\
\hline 5 & $\begin{array}{l}\text { Figura Complexa de } \\
\text { Rey }\end{array}$ & 11 & 17 & Teste & $\begin{array}{l}\text { Organização percetivo- } \\
\text { motora, atenção e } \\
\text { memória visual imediata }\end{array}$ & $\begin{array}{l}\text { Figura A: 5- } \\
\text { Figura B: } 4-8\end{array}$ & $\begin{array}{l}\text { Adaptação, estudos normativos e de validade } \\
\text { (Rey/CEGOC, 1998) }\end{array}$ & Não \\
\hline 6 & SGS-II & 10 & 16 & Testes & $\begin{array}{l}\text { Desenvolvimento/ } \\
\text { Cognitivo }\end{array}$ & $0-5$ & $\begin{array}{l}\text { SGS-II: tradução (Bellman et al./Rocha et } \\
\text { al./CEGOC, 2003); adaptação, estudos normativos } \\
\text { preliminares e estudos de validade (Varajidás, } \\
\text { Machado et al., 2017) }\end{array}$ & Sim \\
\hline 7 & CAT & 9 & 14 & Teste & $\begin{array}{c}\text { Projetivo/ } \\
\text { personalidade }\end{array}$ & $\begin{array}{c}3-10 \\
(\mathrm{CAT}-\mathrm{A}) \\
6-10 \\
(\mathrm{CAT}-\mathrm{H})\end{array}$ & $\begin{array}{l}\text { CAT-A (Fonseca, 1983) e CAT-H (Silva, 1995): } \\
\text { adaptação, estudos normativos e de validade }\end{array}$ & Não \\
\hline 7 & $\begin{array}{l}\text { Teste de Desenho da } \\
\text { Família } \\
\text { - Corman }(n=1)\end{array}$ & 9 & 14 & Teste & Projetivo /personalidade & $5 / 6-$ & Sem estudos em Portugal & Não \\
\hline 9 & $\begin{array}{l}\text { Provas de Diagnóstico } \\
\text { Pré-Escolar (La Cruz) }\end{array}$ & 7 & 11 & Testes & Prontidão escolar & $5-7$ & $\begin{array}{l}\text { Adaptação, estudos normativos e de validade } \\
\text { (LaCruz/Coelho \& Remédio/CEGOC, 1993) }\end{array}$ & Sim \\
\hline 9 & $\begin{array}{l}\text { Escalas do modelo } \\
\text { ASEBA (Achenbach) } \\
\text { - CBCL }(n=3) \\
\text { - TRF }(n=2) \\
\text { - ASEBA }(n=2)\end{array}$ & 7 & 11 & Inventário & $\begin{array}{l}\text { Comportamento } \\
\text { /adaptação } \\
\text { socioemocional }\end{array}$ & $\begin{array}{c}\text { Existe uma } \\
\text { versão } 1 \frac{1}{2} 2-5\end{array}$ & $\begin{array}{l}\text { Adaptação, estudos normativos e de validade } \\
\text { (Achenbach et al./Psiquilibrios, 2014) }\end{array}$ & $\underset{\text { (na versão } 1 \frac{1}{2} 2 \text { a } 5 \text { ) }}{\operatorname{Sim}}$ \\
\hline 9 & $\begin{array}{l}\text { Teste de Desenho da } \\
\text { Figura Humana } \\
\text { - Goodenough-Harris } \\
(n=3)\end{array}$ & 7 & 11 & Teste & Inteligência & $5-12$ & $\begin{array}{l}\text { Técnica de Naglieri: Adaptação, estudos normativos } \\
\text { preliminares e estudos de validade (Veiga, 2001) }\end{array}$ & Não \\
\hline & 2 Escala de Conners & 5 & 8 & Inventário & $\begin{array}{l}\text { Comportamento } \\
\text { /adaptação } \\
\text { socioemocional }\end{array}$ & $6-11$ & $\begin{array}{l}\text { Adaptação, estudos normativos preliminares e } \\
\text { estudos de validade da versão revista (Rodrigues, } \\
\text { 2007) }\end{array}$ & Não \\
\hline & $\begin{array}{l}3 \text { Bateria de Aptidões } \\
\text { para a Aprendizagem } \\
\text { Escolar (BAPAE) }\end{array}$ & 4 & 6 & Testes & Adaptação à escola & $\begin{array}{l}\text { 6- } \\
\left(1^{\circ} \text { ou } 2^{\circ} \text { ano do }\right. \\
\text { ensino básico })\end{array}$ & $\begin{array}{l}\text { Adaptação, estudos normativos e de validade (La } \\
\text { Cruz/Luz \& Remédio/CEGOC, 1993) }\end{array}$ & Não \\
\hline & $\begin{array}{l}3 \text { Teste Apercetivo de } \\
\text { Roberts para Crianças }\end{array}$ & 4 & 6 & Teste & Projetivo/ personalidade & $\begin{array}{c}6-15 \\
6-18(\text { ed. } 2)\end{array}$ & $\begin{array}{l}\text { Adaptação, estudos normativos preliminares (6 a } 9 \\
\text { anos) e estudos de validade (Gonçalves et al., 1999) } \\
2^{\text {a }} \text { Edição: sem estudos em Portugal }\end{array}$ & Não \\
\hline & 3 Era uma vez... & 4 & 6 & Teste & $\begin{array}{c}\text { Projetivo/ } \\
\text { personalidade }\end{array}$ & $5-12$ & $\begin{array}{l}\text { Interpretação baseada na análise qualitativa das } \\
\text { respostas; estudos de validade (Fagulha/CEGOC, } \\
\text { 1997) }\end{array}$ & Não \\
\hline
\end{tabular}

Nota. WISC=Wechsler Intelligence Scale for Children; Griffiths=Escalas de Desenvolvimento Mental de Griffiths; WPPSI=Wechsler Preschool and Primary Scale of Intelligence $; \mathrm{CPM}=$ Coloured Progressive Matrices; $\mathrm{CPM}-\mathrm{P}=$ Coloured Progressive Matrices - Parallel Version $;$ Rey $=$ Teste da Figura Complexa de Rey; SGS=Schedule of Growing Skills; CAT=Children Apperception Test; CAT-A = CAT-Figuras Animais; CAT-H=CATFiguras Humanas; $\mathrm{CBCL}=$ Child Behavior Checklist; TRF=Teacher Report Form; ASEBA=Achenbach System of Empirically Based Assessment.

\section{Discussão}

Esta investigação pretendeu caracterizar os psicólogos portugueses que utilizam testes e outros instrumentos de avaliação psicológica com crianças dos 0 aos 7 anos de idade, do ponto de vista sociodemográfico e da sua formação e contexto de atividade profissional. Procurou-se, igualmente, compreender quais os principais objetivos subjacentes à utilização dos referidos instrumentos e quais os mais usados com esta faixa etária.

Em linha com estudos em Portugal e noutros países (Albritton et al., 2019; Seabra-Santos et al., 2019; Reppold et al., 2000), a grande maioria dos 
inquiridos são mulheres, com uma média de idades entre os 35 e os 40 anos, e com uma duração de atividade profissional maioritariamente entre os 10 e os 15 anos. Também de acordo com o estudo português que tomou uma amostra mais alargada (Seabra-Santos et al., 2019), os participantes têm formação em áreas com maior tradição da prática de avaliação psicológica, sendo a percentagem com formação em Psicologia Clínica e da Saúde muito expressiva (57.1\%), seguindo-se a Psicologia Escolar e da Educação, com uma taxa bastante inferior (20.6\%). Já no que diz respeito ao campo de atuação profissional a distribuição por estas duas áreas é bastante mais equilibrada (42.9\% e $38.1 \%$, respetivamente). Este último resultado contrasta com estudos anteriores que registaram percentagens muito superiores na área da Psicologia Clínica e da Saúde, comparativamente à Psicologia Escolar e da Educação, sendo esta desproporção cerca de o dobro no estudo português (Seabra-Santos et al., 2019) e cerca de cinco vezes superior no estudo congénere conduzido em Espanha (Muñiz et al., 2020). Estes dados sugerem que na avaliação psicológica de crianças mais novas estão bastante presentes os psicólogos que exercem a sua atividade em contexto escolar e pré-escolar. A avaliação/diagnóstico surge como o objetivo mais frequentemente apontado para o uso de testes com crianças dos 0 aos 7 anos, sendo o rastreio psicológico menos referido, o que poderá sugerir, à semelhança do verificado noutros estudos (Albritton et al., 2019), uma menor orientação dos serviços para uma atuação preventiva de identificação precoce de necessidades e de respostas adequadas ou, por outras palavras, um maior foco na caraterização de situações e de casos já sinalizados.

Dos 15 instrumentos mais mencionados, 13 são testes que envolvem diretamente a criança na resolução de problemas ou tarefas. Assim, em contraste com estudos realizados noutros países, nomeadamente em contexto escolar (Benson et al., 2019), os inventários comportamentais aparecem menos representados, apesar de avaliarem uma ampla diversidade de comportamentos adaptativos e/ou disfuncionais em diferentes contextos e com recurso a diferentes informadores, em pouco tempo e envolvendo custos reduzidos (Major \& Seabra-Santos, 2013).
Tendo em conta as especificidades desta faixa etária, que conferem uma importância acrescida ao recurso a outros informadores, como pais e educadores, este dado pode sinalizar que a recolha de informação poderá ser feita através de métodos de avaliação informais, não contemplados neste estudo (e.g., entrevistas não estruturadas).

Dos 15 instrumentos mais referidos pelos participantes, nove têm como foco da avaliação construtos de natureza cognitiva ou desenvolvimental. A referência a dois testes de prontidão escolar e de avaliação de aptidões necessárias para as aprendizagens escolares, usados para apoiar decisões na fase de transição do pré-escolar para o $1^{\circ}$ ciclo, traduz uma preocupação preventiva que importa sublinhar (Albritton et al., 2019).

No conjunto dos instrumentos referidos, as escalas de inteligência de Wechsler (WISC e WPPSI) assumiram um destaque particular, sugerindo a preferência dos psicólogos por uma avaliação do funcionamento cognitivo compreensiva, integradora de funções, processos ou conteúdos, em vez de testes complementares mais específicos (e.g., Figura Complexa de Rey). Esta preponderância das escalas de inteligência de Wechsler foi também encontrada em estudos no contexto forense (LaDuke et al., 2017), neuropsicológico (Rabin et al., 2005) ou escolar (Oakland et al., 2016), sendo estes instrumentos considerados, neste último contexto, como "gold standard" para a avaliação da inteligência dos alunos (Benson et al., 2019). A clara preferência manifestada pelos psicólogos portugueses em relação a estas escalas reforça a necessidade de aferição para a população portuguesa das suas edições mais recentes (WPPSI-IV, publicada nos EUA em 2012, e WISC-V, editada em 2014), recentemente adaptadas e validadas em outros países.

Igualmente à semelhança do que se observa noutros estudos (Oakland et al., 2016), o teste das Matrizes Progressivas de Raven surge também como um dos mais utilizados, sendo uma alternativa de medida cognitiva, não-verbal, com a vantagem de ser de aplicação rápida e exigir poucos recursos, para além de ser o único dos testes referidos que pode ser administrado coletivamente. Note-se que a forma colorida deste teste, aplicada a crianças dentro da faixa etária em estudo, se encontra aferida para a população 
portuguesa (Simões, 2000; Forma paralela: CEGOC, 2009).

Mesmo não havendo ainda normas nacionais publicadas, a Griffiths aparece como a escala de desenvolvimento psicológico mais utilizada pelos psicólogos desta subamostra, o que pode sugerir uma utilização das tarefas no quadro de avaliações mais compreensivas do que normativas do funcionamento e habilidade cognitiva. Desta escala existe já disponível a tradução da versão III, da qual estão em curso estudos de validação para a população portuguesa (https://www.hogrefe.com/pt/).

A menor ênfase em instrumentos de avaliação socio-emocional (personalidade), que representam pouco mais de um terço das medidas referidas, poderá traduzir a necessidade de mais ferramentas para avaliar estes construtos em crianças desta faixa etária. A publicação, num futuro próximo, das Escalas de Comportamento para a Idade PréEscolar - $2^{\mathrm{a}}$ edição (PKBS-2) poderá contribuir para colmatar esta lacuna, na medida em que se trata de um inventário que avalia as aptidões sociais e os problemas de comportamento de crianças em idade pré-escolar, em contexto casa e escola (Hogrefe, 2020; Major \& Seabra-Santos, 2014). Note-se que, dos seis instrumentos mencionados para avaliar a área socio-emocional (personalidade), quatro são testes projetivos, o que vai ao encontro da ideia de Piotrowski (2019) segundo a qual o estado futuro das técnicas projetivas na avaliação psicológica pode ser limitado, mas não está "moribundo". Aliás, os nossos dados não estão em sintonia com os estudos que apontam para a diminuição do uso de tais técnicas na avaliação psicológica em contexto escolar (Benson et al., 2019; Oakland et al., 2016).

Comparando os resultados encontrados nesta investigação com os do inquérito anterior mais recente realizado em Portugal (Diniz et al., 2007), verificamos um elevado grau de sobreposição no que toca aos instrumentos mais utilizados. Assim, se nos cingirmos àqueles que são aplicáveis a crianças dos 0 aos 7 anos, o estudo de Diniz e colaboradores ressalta a WISC (nas suas várias edições), as Matrizes de Raven (nas suas diversas versões) e a Figura Complexa de Rey como as provas mais frequentes para avaliar o domínio cognitivo. Também nesse estudo a WISC é mencionada cerca do dobro das vezes em comparação com o instrumento que se lhe segue na lista de preferência dos utilizadores. $\mathrm{O}$ teste do Desenho da Figura Humana de Goodenough e a WPPSI-R estão, igualmente, presentes entre as provas mais usadas. A escala de Griffiths surge como o instrumento a que os psicólogos mais recorrem na avaliação do desenvolvimento, enquanto o CAT e o Desenho da Família figuram como instrumentos muito referidos (cf. provas de personalidade e provas expressivas, respetivamente).

Esta continuidade nos instrumentos de avaliação psicológica a que os psicólogos recorrem ao longo do tempo também foi constatada noutros estudos, sobretudo no que diz respeito à avaliação cognitiva (Benson et al., 2019). Esta estabilidade pode, em parte, ser explicada pelo facto de os psicólogos se sentirem confortáveis com testes com os quais estão familiarizados, em muitos casos porque aprenderam a utilizá-los no decurso da sua formação académica (Albritton et al., 2019). Por outro lado, a variedade de recursos disponíveis é limitada, não estando aferidos para o nosso país alguns dos instrumentos relevantes ou havendo dificuldades financeiras que inibem a aquisição de novos testes por parte das instituições. Acresce que também não existe, no nosso país, qualquer autoridade que obrigue à descontinuação do uso de um teste, face à publicação de uma nova edição. Neste contexto, aliás, o elevado custo dos testes, destacado como dificuldade por alguns dos participantes, poderá explicar a reprodução nãoautorizada de materiais por parte dos psicólogos, situação ainda bastante presente no nosso país (Seabra-Santos et al., 2019), a sugerir a necessidade de um papel mais ativo das instituições competentes na promoção e controlo das boas práticas no uso de testes (Almeida \& Cruz, 1998; Muñiz et al., 2001). A questão económica poderá, igualmente, ser uma das justificações para a opção por provas gráficas de desenho (Desenho da Figura Humana, Desenho da Família), que podem estar disponíveis sem custos (Oakland et al., 2016). Por outro lado, esta estabilidade traduz-se na fraca utilização de instrumentos mais recentes. Neste contexto, é de notar a ausência de referências à Bateria de Avaliação Neuropsicológica de Coimbra (BANC), apesar de se tratar de um instrumento aplicável a partir dos 5 anos de idade e que possui normas 
recentes para a população portuguesa (Simões et al., 2016). Esta omissão é no entanto compreensível considerando o reduzido intervalo temporal entre a data da edição da bateria (dezembro de 2016) e a resposta ao presente inquérito (2018).

Este estudo coloca em evidência algumas fragilidades em muitos dos instrumentos usados na avaliação de crianças mais novas, seja porque não foram criados para estas idades (englobam leques etários alargados ou constituem extensões de outros que foram desenvolvidos para avaliar crianças mais velhas, como é o caso da WPPSI-R ou da versão $1 \frac{1}{2} 2-5$ anos do modelo ASEBA), ou tendo em conta que se encontram pouco suportados por estudos normativos e/ou de validade atualizados para as crianças portuguesas desta faixa etária. Destaca-se, igualmente, a ausência de referências a entrevistas estruturadas ou grelhas de observação direta, o que aponta para a necessidade de reforçar a investigação e formação sobre este tipo de técnicas e instrumentos, no sentido de viabilizar processos de avaliação psicológica assentes numa abordagem multi-método e multiinformador (Benson et al., 2019).

Apesar de haver um consenso alargado quanto à importância dos primeiros anos de vida para a identificação precoce de fatores de risco que permitam uma intervenção atempada, no sentido de prevenir ou minorar problemas no futuro, pouca atenção tem sido dada a esta faixa etária mais nova, do ponto de vista da avaliação psicológica (Major \& Seabra-Santos, 2014). Aliás, esta situação é verificada também por outros autores, que destacam um menor recurso a instrumentos de avaliação psicológica com crianças antes da entrada na escola (Benson et al., 2019; Oakland, 2009), ou uma menor formação para atender às necessidades específicas destas idades (Albritton et al., 2019).

Este estudo apresenta algumas limitações que importa referir, no sentido de que poderão apontar caminhos para investigações a realizar no futuro. Em grande parte, estas limitações estão relacionadas com o facto de o estudo se inserir numa investigação mais ampla, que contemplou o uso de instrumentos de avaliação psicológica com pessoas de todas as idades. Uma primeira limitação diz respeito à delimitação das faixas etárias que constam no questionário e, nomeadamente, ao facto de as idades consideradas entrarem já na idade escolar (6-7 anos). A inclusão destas idades, decorrente do esforço de conciliação com as realidades dos outros países envolvidos, excluiu a possibilidade de se obter uma ideia mais "pura" sobre as especificidades da avaliação psicológica antes da entrada na escola. Por outro lado, ainda que tenham sido selecionadas para análise as respostas dos participantes que centram as atividades de avaliação maioritariamente em crianças dos 0 aos 7 anos, o facto de não se dedicarem em exclusivo a esta faixa etária excluiu a possibilidade de identificar, nas respetivas respostas, quais os instrumentos especificamente usados com crianças destas idades. Também não foi viável analisar separadamente as respostas correspondentes às faixas etárias de 0 a 3 anos e de 3 a 7 anos, dado que a quase totalidade dos participantes incluídos na primeira categoria, também pertenciam à segunda.

Estudos futuros poderão focar-se mais especificamente nos psicólogos portugueses que trabalham com crianças antes da entrada na escola, procurando identificar e distinguir com maior precisão de que forma são concretizadas as tarefas de avaliação psicológica nesta faixa etária. Será interessante compreender quais as necessidades sentidas por estes profissionais no que diz respeito à avaliação psicológica, nomeadamente no que toca à disponibilidade de instrumentos validados, à formação específica que possuem para trabalhar com crianças pequenas e aos principais desafios encontrados (Albritton et al., 2019). Estes pontos reforçam a necessidade de maior investigação nesta área e de fortalecer a formação de psicólogos, no sentido de desenvolver respostas de qualidade e direcionadas às necessidades e características específicas das crianças nos primeiros anos de vida (Albritton et al., 2019). Com efeito, a avaliação psicológica nestas idades é um trabalho muito mais amplo e complexo do que a mera aplicação de instrumentos, sendo a perícia do psicólogo que confere valor ao processo (Bracken, 1994), ou seja, o examinador deve ser melhor do que os testes que utiliza (Fletcher-Janzen, 2009; Kaufman, 1979, 1994). Por outras palavras, “ $A$ fool with a tool is still a fool" (frase atribuída a Grady Booch, s.d.). 


\section{Referências}

Achenbach T. M., Rescorla, L. A., Dias, P., Ramalho, V., Lima, V. S., Machado, B. C., \& Goçalves, M. (2014). Manual do Sistema de Avaliação Empiricamente Validado (ASEBA) para o período pré-escolar e escolar: Um sistema integrado de avaliação com múltiplos informadores. Psiquilibrios Edições.

Albritton, K., Mathews, R. E., \& Boyle, S. G. (2019). Is the role of the school psychologist in early childhood truly expanding? A national survey examining school psychologists' practices and training experiences. Journal of Applied School Psychology, 31(1), 1-19. https://doi.org/10.1080/15377903.2018.1462280

Almeida, L. S., \& Cruz, O. (1985). A utilização dos testes psicológicos: Resultados de um inquérito junto de psicólogos portugueses. Jornal de Psicologia, 4(5), 11-18.

Almeida, L. S., Simões, M. R., Seabra-Santos, M. J., \& Almiro, P. A. (2018, Setembro). Práticas de avaliação psicológica em Portugal: Resultados gerais de inquérito realizado sobre a utilização de testes e outros instrumentos. Conferência plenária apresentada no $10^{\circ}$ Congresso da Associação Iberoamericana de Diagnóstico e Avaliação Psicológica (AIDAP), Coimbra.

Almeida, S., \& Cruz, O. (1998). O uso dos testes como revelador de modelos de prática psicológica: Estudo junto dos psicólogos portugueses. Psicologia: Teoria e Pesquisa, 4(3),207-224.

American Educational Research Association, American Psychological Association, American Council on Measurement in Education. (2014). Standards for educational and psychological testing ( ${ }^{\text {rd }}$ ed.). https://www.testingstandards.net/uploads/7/6/ 6/4/76643089/standards_2014edition.pdf

Archer, R. P., Wheeler, E. M A., \& Vauter, R. A. (2016). Empirically supported forensic assessment. Clinical Psychology Science and Practice, 23(4), 348-364. https://doi.org/10.1111/cpsp.12171

Barbarin, O. A. (2007). Mental health screening of preschool children: Validity and reliability of ABLE. American Journal of
Orthopsychiatry, 77(3),

$402-418$ https://doi.org/10.1037/0002-9432.77.3.402

Bellman, S. Lingam, \& A. Aukett (2003). SGS-II - Escala de Avaliação das Competências no Desenvolvimento Infantil: Manual técnico. CEGOC.

Benson, N. F., Floyd, R. G., Kranzler, J. H., Eckert, T. L., Fefer, S. A., \& Morgan, G. B. (2019). Test use and assessment practices of school psychologists in the United States: Findings from 2017 national survey. Journal of School Psychology, 72, 29-48. https://doi.org/10.1016/j.jsp.2018.12.004

Borges, P., Costa, I. P., Ferreira, C. T., Gil, I. C., Carvalhão, I., Fernandes, S., \& Veríssimo, M. (2012). Escalas de Desenvolvimento Mental de Ruth Griffiths: Adaptação para a população portuguesa. In L. Mata, F. Peixoto, J. Morgado, J. C. Silva \& V. Monteiro (Eds.), Actas do $12 .^{\circ}$ Colóquio Internacional de Psicologia e Educação (pp. 922-932). ISPA.

Bracken, B. A. (1994). Advocating for effective preschool assessment practices: A comment on Bagnato and Neisworth. School Psychology Quarterly, 9(2), 103-108.

Breitenstein, S. M., Hill, C., \& Gross, D. (2009). Understanding disruptive behavior problems in preschool children. Journal of Pediatric Nursing, 24(1), 3-12. https://doi.org/10.1016/j.pedn.2007.10.007

Carvalho, L., Almeida, I. C., Felgueiras, I., Leitão, S., Boavida, J., Santos, P. C., Serrano, A., Brito, A. T., Lança, C., Pimentel, J. S., Pinto, A. I., Grande, C., Brandão, T., \& Franco, V. (2016). Práticas recomendadas em intervenção precoce na infância: Um guia para profissionais. Associação Nacional de Intervenção Precoce.

Casullo, M. M. (2009). La evaluación psicológica: Modelos, técnicas y contextos. Revista Iberoamericana de Diagnóstico y Evaluación Psicológica, 27(1), 9-28.

https://www.aidep.org/sites/default/files/articl es/R07/R077.pdf

Center on the Developing Child at Harvard University. (2017). Three principles to improve outcomes for children and families. http://www.developingchild.harvard.edu

Cohen, R. J., \& Swerdlik, M. E. (2018). Psychological testing and assessment: An 
introduction to tests and measurement $\left(9^{\text {th }}\right.$ ed.). McGraw-Hill Education.

Cruz, O., \& Almeida, L. S. (1987). A utilização dos testes psicológicos em Portugal: Análise segundo a formação e o domínio de prática profissional. Jornal de Psicologia, 6(1), 1319.

Diniz, A. M., Almeida, L. S., \& Pais, L. G. (2007). Contextos profissionais e práticas de avaliação psicológica: Inquérito aos psicólogos portugueses. Psico-USF, 12(1), 112.

http://dx.doi.org/10.1590/S141382712007000100002

Evers, A., McCormick, C. M., Hawley, L. R., Muñiz, J., Balboni, G., Bartram, D., Boben, D., Egeland, J., El-Hassan, K., FernándezHermida, J. R., Fine, S., Frans, Ö., Gintiliené, G., Hagemeister, C., Halama, P., Iliescu, D., Jaworowska, A., Jiménez, P., Manthouli, M., ... Zhang, J. (2017). Testing practices and attitudes toward tests and testing: An international survey. International Journal of Testing, 17(2), 158-190. https://doi.org/10.1080/15305058.2016.12164 34

Evers, A., Muñiz, J., Bartram, D., Boben, D., Egeland, J., Fernández-Hermida, J. R., Frans, Ö., Gintiliené, G., Hagemeister, C., Halama, P., Iliescu, D., Jaworowska, A., Jiménez, P., Manthouli, M., Matesic, K., Schittekatte, M., Sümer, H. C., \& Urbánek, T. (2012). Testing practices in the 21st Century: Developments and European psychologists' opinions. European Psychologist, 17(4), 300-319. https://doi.org/10.1027/1016-9040/a000102

Fagulha, T. (1997). Manual: Era uma vez. CEGOC.

Fletcher-Janzen, E. (2009). Intelligent testing: Bridging the gap between classical and romantic science in assessment. In J. C. Kaufman (Ed.), Intelligent testing: Integrating psychological theory and clinical practice. A tribute to Alan S. Kaufman (pp. 15-29). Cambridge University Press.

Fonseca, A. C. (1983). O estudo de temas dominantes ou banais no CAT numa população portuguesa de 6 anos de idade [Provas de Aptidão Pedagógica e de Capacidade Científica não publicadas].
Faculdade de Psicologia e de Ciências da Educação, Universidade de Coimbra.

Fontaine, J. (2017). Welcome to the ECPA14. In P. Ferreira, A. Ferreira, I. Afonso, \& A. M. Simão (Eds.), ECPA14 - 14th European Conference on Psychological Assessment: Book of abstracts (p. iv). Faculdade de Psicologia, Universidade de Lisboa.

Gonçalves, M., Morais, A. P., Pinto, H., \& Machado, C. (1999). Teste Aperceptivo de Roberts para Crianças. In M. R. Simões, M. Gonçalves, \& L. S. Almeida (Eds.), Testes e provas psicológicas em Portugal (Vol. 2, pp. 185-198). APPORT/ SHO.

Griffiths, R., \& Huntley, M. (2007). Manual: Escala de Desenvolvimento Mental de Griffiths (dos 0 aos 2 anos). CEGOC.

Griffiths, R., Luiz, D. et al. (2007). Manual técnico: Escala de Desenvolvimento Mental de Griffiths (extensão revista dos 2 aos 8). CEGOC.

Hogrefe. (2020). Catálogo 2020. https://issuu.com/editorahogrefe/docs/webcata_logo-hogrefe-25mar2020

International Test Commission. (2013). International guidelines for test use. https://www.intestcom.org/files/guideline_test _use.pdf

International Test Commission. (2017). The ITC guidelines for translating and adapting tests ( $2^{\text {nd }}$ ed. $)$.

https://www.intestcom.org/files/guideline_test _adaptation_2ed.pdf

Kaufman, A. S. (1979). Intelligent testing with the $W I S C$ - $R$. Wiley.

Kaufman, A. S. (1994). Intelligent testing with the WISC-III. Wiley.

La Cruz (1993). Bateria de aptidões para a aprendizagem escolar. CEGOC.

La Cruz (1993). Manual: Pré-escolar. CEGOC.

LaDuke, C., Barr, W., Brodale, D. L., \& Rabin, L. A. (2018). Toward generally accepted forensic assessment practices among clinical neuropsychologists: A survey of professional practice and common test use. The Clinical Neuropsychologist, $\quad 32(1), \quad$ 145-164. http://doi.org/10.1080/13854046.2017.134671 1

Major, S., \& Seabra-Santos, M. J. (2013). Uso de inventários comportamentais para a avaliação 
socioemocional em idade pré-escolar. Avaliação Psicológica, 12(1), 101-107. http://pepsic.bvsalud.org/pdf/avp/v12n1/v12n 1a13.pdf

Major, S., \& Seabra-Santos, M. J. (2014). Preschool and Kindergarten Behavior Scales Second Edition (PKBS-2): Adaptação e estudos psicométricos da versão portuguesa. Psicologia: Reflexão e Crítica, 27(4), 689-699. http://dx.doi.org/10.1590/1678-

7153.201427409

Muñiz, J., Bartram, D., Evers, A., Boben, D., Matesic, K., Glabeke, K., FernándezHermida, J. R., \& Zaal, J. N. (2001). Testing practices in European Countries. European Journal of Psychological Assessment, 17(3), 201-211. https://doi.org/10.1027/1016-9040/a000102

Muñiz, J., Hernández, A., \& Fernández-Hermida, J. R. (2020). Utilización de los test en España: El punto de vista de los psicólogos. Papeles del Psicólogo, 41(1), 1-15. https://doi.org/10.23923/pap.psicol2020.2921

Muñiz, J., Prieto, G., Almeida, L., \& Bartram, D. (1999). Test use in Spain, Portugal and Latin American Countries. European Journal of Psychological Assessment, 15(2), 151-157. https://doi.org/10.1027//1015-5759.15.2.151

National Scientific Council on the Developing Child. (2004). Children's emotional development is built into the architecture of their brains: Working Paper No. 2. http://www.developingchild.net

Oakland, T, Douglas, S., \& Kane, H. (2016). Top 10 standardized tests used internationally with children and youth by school psychologists in 64 countries: A 24-year follow-up study. Journal of Psychoeducational Assessment, 34(2), 166-176. https://doi.org/10.1177/0734282915595303

Oakland, T. (2009). How universal are test development and use? In E. Grigorenko (Ed.), Assessment of abilities and competencies in an era of globalization (pp. 1-40). Springer.

Ordem dos Psicólogos Portugueses. (2011). Código deontológico. https://www.ordemdospsicologos.pt/pt/cod_de ontologico
Ordem dos Psicólogos Portugueses. (2018). Conselho Consultivo para a Acreditação de Testes e Provas Psicológicas.

https://www.ordemdospsicologos.pt/pt/comiss oes/comissao/index/conselho-consultivo-paraa-acreditacao-de-testes-e-provas-psicologicasda-opp

Piotrowski, C. (2017). Neuropsychological testing in professional psychology specialties: Summary findings of 36 studies (1990-2016) in applied settings. Journal of the Indian Academy of Applied Psychology, 43(1), 134144.

Piotrowski, C. (2019). Projective techniques are not moribund: Comment on the Benson et al. (2019) assessment practices article [Editorial]. SIS Journal of Projective Psychology and Mental Health, 26, 73-76.

Prieto, G., \& Muñiz, J. (2000). Un modelo para evaluar la calidad de los tests utilizados en España. Papeles del Psicólogo, 77, 65-75.

Rabin, L. A., Barr, W. B., \& Burton, L. A. (2005). Assessment practices of clinical neuropsychologists in the United States and Canada: A survey of INS, NAN, and APA Division 40 members. Archives of Clinical Neuropsychology, 20, 33-65. https://doi.org/10.1016/j.acn.2004.02.005

Raven, J., Raven, J. C., \& Court, J. H. (2009). Manual CPM-P: Matrizes Progressivas Coloridas (Forma Paralela). CEGOC.

Reppold, C. T., Wechsler, S. M., Almeida, L. S., Elosua, P., \& Hutz, C. S. (2020). Perfil dos psicólogos brasileiros que utilizam testes psicológicos: Áreas e instrumentos utilizados. Psicologia: Ciência e Profissão, 40, 1-14. http://doi.org/10.1590/1982-3703003201348

Rey, A. (1998). Teste de Cópia de Figuras Complexas. CEGOC.

Ricou, M., Cordeiro, S. A., Franco, A. R., \& Costa-Lobo, C. (2018). The identity of Psychology. The Psychologist: Practice and Research Journal, 1(1) 1-15. https://doi.org/10.33525/pprj.v1i1.25

Rodrigues, A. N. (2007). Escalas de Conners Revistas (Conners Rating Scales Revised, 1997): Formas Reduzidas para Pais e Professores. In M. R. Simões, C. Machado, M. M. Gonçalves, \& L. S. Almeida (Eds.), Avaliação Psicológica: Instrumentos 
validados para a população portuguesa (Vol. 3, pp. 203-227). Edições Quarteto.

Seabra-Santos, M.J., Almiro, P.A., Simões, M.R., \& Almeida, L.S. (2019). Testes psicológicos em Portugal: Atitudes, problemas e perfil dos utilizadores. Revista Iberoamericana de Diagnóstico y Evaluación - e Avaliação Psicológica, 101-112. https://doi.org/10.21865/RIDEP53.4.08

Silva, D. R. (1995). Teste de Apercepção para Crianças (Figuras Humanas): CAT-H. In L. S. Almeida, M. R. Simões, \& M. M. Gonçalves (Eds.), Provas Psicológicas em Portugal (pp. 321-329). APPORT.

Simões, M. R. (2000). Investigações no âmbito da Aferição Nacional do Teste das Matrizes Progressivas Coloridas de Raven (M.P.C.R). Fundação Calouste Gulbenkian/Fundação para a Ciência e a Tecnologia.

Simões, M. R., Albuquerque, C. P., Pinho, M. S., Vilar, M., Pereira, M., Lopes, A. F., SeabraSantos, M.J., Alberto, I., Lopes, C., Martins, C., \& Moura, O. (2016). Bateria de Avaliação Neuropsicológica de Coimbra (BANC): Manual técnico. CEGOC.

Stroud, L., Foxcroft, C., Green, E., Bloomfield, S., Cronje, J., Hurter, K., Lane, H., Marais, R., Marx, C., McAlinden, P., O'Connell, R., Paradice, R., \& Venter, D. (2018). Griffiths III - Escalas de Desenvolvimento Mental de Griffiths - $3^{a}$ edição: Manual técnico. Hogrefe.

Varajidás, C. A., Machado, M., Mota, M. P., Martins, R., Lisboa, M. C., Soares, I., Sousa, S., \& Leitão, J. C. (2017). Propriedades psicométricas da escala de avaliação das competências no desenvolvimento infantil II Schedule of Growing Skills II (SGS II): Versão Portuguesa. Psychologica, 60(1), 7-18. https://doi.org/10.14195/1647-8606_60-1_1

Veiga, I. S. (2001). Estudos com o Teste do Desenho da Figura Humana de Jack Naglieri: Draw-a-Person (DAP:QSS): Sistema de avaliação quantitativo [Tese de Mestrado não publicada]. Universidade de Coimbra, Faculdade de Psicologia e de Ciências da Educação, Coimbra.

Washington State Office of Superintendent of Public Instruction. (2008). A guide to assessment in early childhood: Infancy to age eight.

http://www.k12.wa.us/earlylearning/pubdocs/ assessment_print.pdf

Wechsler, D. (2003a). Escala de Inteligência de Wechsler para a Idade Pré-Escolar e Primária - Edição Revista: Manual. CEGOC.

Wechsler, D. (2003b). Escala de Inteligência de Wechsler para Crianças - Terceira Edição: Manual. CEGOC.

Wright, C. V., Beattie, S. G., Galper, D. I., Church, A. S., Bufka, L. F., Brabender, V.M., \& Smith, B. L. (2017). Assessment practices of professional psychologists: Results of a national survey. Professional Psychology: Research and Practice, 48(2), 73-78. https://doi.org/10.1037/pro0000086 